\title{
Approximate energy expressions for confining polynomial
}

\section{potentials}

\author{
A. Nanayakkara* and V. Bandara \\ Institute of Fundamental Studies, Hanthana Road, Kandy, Sri Lanka
}

\begin{abstract}
Recently developed asymptotic energy expansion (AEE) method is applied to obtain asymptotic energy expansions (AEEs) of general polynomial potentials. These expansions contain coefficients of the polynomial potentials explicitly. The asymptotic expansions produce very accurate eigen energies. Recurrence relations derived here can be used to obtain asymptotic expansions of polynomial potentials of any degree. Energy eigen value expressions are presented for the 4th, 6th, 8th and 10th degree polynomial potentials. The expansions obtained with AEE method for polynomial potentials have resemblance with WKB expressions obtained by Bender et $\mathrm{al}^{1}$ for the potentials $V(x)=x^{N}(N$ even).
\end{abstract}

\section{INTRODUCTION}

There have been great interest in anharmonic oscillator potentials due to their usefulness in quantum field theory, nuclear physics, particle physics, solid-state physics, and atomic and molecular physics. Both perturbative and non-perturbative methods $^{2-15}$ have been used in the literature to study these potentials with varying success. In this paper, first, we study general polynomial potentials of even degree with recently developed AEE method ${ }^{16}$ and then explicit expansions are derived for the fourth, sixth, eighth, and tenth degree polynomial potentials. The asymptotic eigen energy expansions are written as

$\sum_{k} b_{k} E_{n}^{\alpha_{k}}=\left(n+\frac{1}{2}\right) \hbar$

*Corresponding author (E-mail: asiri@ifs.ac.lk) 
where $E_{n}^{\alpha_{k}}$ is the $\alpha_{k}$ th power of the eigen energy, corresponding to $n t h$ excited state and coefficients $b_{k}{ }^{\prime} s$ are known explicitly as polynomials in coefficients of the polynomial potential. The AEE method is well suited for polynomial potentials because they have the property

$V(x)=\sum_{k=0}^{N} V_{k}(x)$

and

$V_{k}(\lambda x)=\lambda^{n}{ }^{k} V_{k}(x)$

and which is required by AEE method. The quantity $J(E)$ is defined as

$$
J(E) \equiv \frac{1}{2 \pi} \int_{\gamma} P(x, E) d x
$$

with the quantization condition $J(E)=n \hbar . P(x, E)$ in (3) satisfies the equation

$$
\frac{\hbar}{i} \frac{\partial P(x, E)}{\partial x}+P^{2}(x, E)=E-\sum_{k=0}^{N} V_{k}(x)=P_{C}(x, E)
$$

The contour $\gamma$ encloses two physical turning points of $P_{C}(x, E)$. In order to obtain AEE, first $P(x, E)$ is expanded in a series of powers of energy $E$. By evaluating (3) and then applying quantization condition $J(E)=n \hbar$, the AEE is obtained. Main aim of this paper is to present explicit AEE for even degree polynomial potentials.

The outline of the paper is as follows. Recurrence relations for even degree general polynomial potentials are derived in section 2. Also a general method of obtaining asymptotic energy expansions (AEEs) is described there. In section 3, AEEs are obtained for the fourth degree polynomial potentials with arbitrary coefficients. Asymptotic expansions for the 6th, 8th and 10th degree polynomial potentials are presented in the section 4 .

\section{GENERAL POLYNOMIAL POTENTIALS AND RECURRENCE RELATIONS}

First we consider the one dimensional polynomial potential of degree $2 \mathrm{~N}$

$$
V(x)=x^{2 N}+\beta_{1} x^{2 N-1}+\ldots \ldots . . \beta_{2 N-1} x .
$$

It is easy to see that $V(x)$ satisfies the condition in (1) with

$$
V_{0}(x)=x^{2 N}, V_{1}(x)=\beta_{1} x^{2 N-1}, \ldots \ldots . . V_{k}(x)=\beta_{k} x^{2 N-k} \ldots . . V_{2 N-1}(x)=\beta_{2 N-1} x
$$

Hence the equation (4) becomes 
$\frac{\hbar}{i} \frac{\partial P(x, E)}{\partial x}+P^{2}(x, E)=E-x^{2 N}-\sum_{k=1}^{2 N-1} \beta_{k} x^{2 N-k}$

Let $\varepsilon=E^{-\frac{1}{2 N}}, y=\varepsilon x$. Then equation (6) becomes, after simplification,

$$
\frac{\hbar}{i} \varepsilon^{2 N+1} \frac{\partial P(y, \varepsilon)}{\partial y}+\varepsilon^{2 N} P^{2}(y, \varepsilon)=1-y^{2 N}-\sum_{k=1}^{2 N-1} \beta_{k} \varepsilon^{k} y^{2 N-k}
$$

Now we expand $P(y, \varepsilon)$ as a power series in $\varepsilon$

$$
P(y, \varepsilon)=\varepsilon^{s} \sum_{m=0} a_{m}(y) \varepsilon^{m}
$$

where s and $a_{m}$ s are determined below. Substituting (8) in equation (7) and equating coefficients of $\varepsilon^{0}$, we get

$$
\begin{aligned}
& s=-N \text { and } a_{0}=\sqrt{1-y^{2 N}} \text {. Then equation (7) becomes } \\
& \sum_{m=0}^{\infty} \frac{\hbar}{i} \frac{d a_{m}}{d y} \varepsilon^{m+1+N}+\sum_{i=0}^{\infty} \sum_{j=0}^{\infty} a_{i} a_{j} \varepsilon^{i+j}=1-y^{2 N}-\sum_{k=1}^{2 N-1} \beta_{k} \varepsilon^{k} y^{2 N-k}
\end{aligned}
$$

Equating coefficients of $\varepsilon^{n}$ for arbitrary $n$ produces three types of recurrence relations. They are

$$
\begin{aligned}
& \text { If } n \leq N \quad a_{n}=-\frac{1}{2 a_{0}}\left\{\beta_{n} y^{2 N-n}+\sum_{i=1}^{n-1} a_{i} a_{n-i}\right\} \\
& \text { If }(2 N-1) \geq n>N \quad a_{n}=-\frac{1}{2 a_{0}}\left\{\beta_{n} y^{2 N-n}+\sum_{i=1}^{n-1} a_{i} a_{n-i}+\frac{\hbar}{i} \frac{d a_{n-N-1}}{d y} .\right.
\end{aligned}
$$

If $n>(2 N-1) \quad a_{n}=-\frac{1}{2 a_{0}}\left\{\sum_{i=1}^{n-1} a_{i} a_{n-i}+\frac{\hbar}{i} \frac{d a_{n-N-1}}{d y}.\right\}$

By using equation (10) with $a_{0}=\sqrt{1-y^{2 N}}, a_{n}$ can be obtain for any $n$ (as in the case of WKB), recursively with computer algebra packages such as MATHEMATICA or MAPLE 
Now $J(\varepsilon)$ becomes

$$
J(\varepsilon)=\frac{\varepsilon^{-N-1}}{2 \pi} \sum_{m=0} \varepsilon^{m} \int_{\gamma_{y}} a_{m}(y, \varepsilon) d y
$$

where $\gamma_{y}$ encloses the two branch points of $\sqrt{1-x^{2 N}}$ (i.e. +1 and -1) on the real axis. By applying the condition that $J(\varepsilon)=n \hbar$, asymptotic expansion of energy can be obtained as

$$
n \hbar=\sum_{m=0} b_{m} \varepsilon^{m-N-1} \quad \text { or } \quad n \hbar=\sum_{m=0} b_{m} E^{-\frac{m-N-1}{2 N}}
$$

where $b_{m}=\frac{1}{2 \pi} \int_{\gamma_{v}} a_{m}(y) d y$

For polynomial potential of even degree $b_{m}$ has one of the two forms given below.

$b_{m}=g\left(\alpha_{i}, N, n, m\right) \eta_{k}(n, m)$ or $b_{m}=h\left(\alpha_{i}, N, n, m\right) \xi_{k}(n, m)$ with

$$
\eta_{N}(n, m)=\int_{\gamma} \frac{y^{n}}{\left(1-y^{2 N}\right)^{m+\frac{1}{2}}} d y, \quad \xi_{N}(n, m)=\int_{\gamma} \frac{y^{n}}{\left(1-y^{2 N}\right)^{m}} d y
$$

where $g\left(\alpha_{i}, N, n, m\right)$ and $h\left(\alpha_{i}, N, n, m\right)$ are known explicitly from the recurrence relations and $\gamma_{y}$ encloses the two branch points of $\sqrt{1-y^{2 N}}$ (i.e. +1 and -1 ) on the real axis. $\eta_{N}(n, m)$ can be written in terms of $\Gamma$ functions as

$\eta_{2 N}(n, m)=\frac{\Gamma\left(\frac{1}{2}-m\right) \Gamma\left(1+\frac{1}{2 N}+\frac{n}{2 N}\right)}{\pi(1+n) \Gamma\left(\frac{1}{2}+\frac{1}{2 N}-m+\frac{n}{2 N}\right)}$

For polynomial potentials of even degree, except $\xi_{2 N}(2 N-1,1)$, all the other terms, containing $\xi_{N}(n, m)$, give zero contribution. In this section we have derived general asymptotic expansions which can be used to obtain AEE for polynomial potential of any even degree. The first few terms of the expansion normally gives very accurate eigen energies when the magnitude of the coefficients of the polynomial potential are less than unity.

\section{FOURTH DEGREE POLYNOMIAL POTENTIALS}

In this section we consider general 4th degree polynomial potentials. The general fourth degree potential is given by $V(x)=x^{4}+a x^{3}+b x^{2}+c x$. 
where $a, b$, and $c$ are real constants. This potential has been treated in literature mainly in its symmetric version; i.e. $a=c=0$. In addition to the symmetric version, here we consider cases when $a \neq 0, b \neq 0, \& c=0$ and $a \neq 0, b \neq 0, \& c \neq 0$ as well.

In order to obtain AEEs let $\varepsilon=E^{-\frac{1}{4}}$ and $y=\varepsilon x$. Using recurrence relation in (10), the explicit expressions of $a_{k}(y) \mathrm{s}$ are found. Then the asymptotic energy expansion becomes

$n \hbar=\sum_{m=0} b_{m} E^{-\frac{m-3}{4}}$

where $b_{m}$ s are given by (13). When $a \neq 0, b \neq 0 \& c \neq 0$, terms in the AEE are lengthier than in the symmetric case. Therefore we consider three cases $(a=0, b \neq 0 \& c=0),(a \neq 0, b \neq 0, \& c=0)$ and $(a \neq 0, b \neq 0, \& c \neq 0)$ separately.

Case I $\quad a=c=0, \quad b \neq 0 \quad$ (Symmetric version: $V(x)=x^{4}+b x^{2}$ )

The AEE for this potential is approximated by the fourteen terms

$$
J(E)=n \hbar \approx-\frac{\hbar}{2}+\sum_{k=0}^{14} b_{k} E^{\left(\frac{3}{4}-\frac{k}{2}\right)}
$$

where

$$
\begin{aligned}
& \Xi=\frac{\Gamma\left[\frac{1}{4}\right]}{\sqrt{\pi} \Gamma\left[\frac{3}{4}\right]} \quad \Omega=\frac{\Gamma\left[\frac{3}{4}\right]}{\sqrt{\pi} \Gamma\left[\frac{1}{4}\right]} \\
& b_{0}=\frac{1}{3} \Xi, \quad b_{1}=-b \Omega, \quad b_{2}=\frac{b^{2}}{32} \Xi, \quad b_{3}=-\left(\frac{b^{3}}{32}+\frac{\hbar^{2}}{4}\right) \Omega \text {, } \\
& b_{4}=-\left(\frac{5 b^{4}}{6144}+\frac{b \hbar^{2}}{192}\right) \Xi, \quad b_{5}=\left(\frac{21 b^{5}}{10240}+\frac{5 b^{2} \hbar^{2}}{128}\right) \Omega, \\
& b_{6}=\left(\frac{5 b^{6}}{65536}+\frac{5 b^{3} \hbar^{2}}{2048}+\frac{11 \hbar^{4}}{1536}\right) \Xi, b_{7}=-\left(\frac{7 b\left(b^{3}+40 \hbar^{2}\right)\left(11 b^{3}+80 \hbar^{2}\right)}{327680}\right) \Omega \text {, } \\
& b_{8}=-\frac{\left(b^{2}\left(585 b^{6}+38080 b^{3} \hbar^{2}+620032 \hbar^{4}\right)\right.}{58720256} \Xi \\
& b_{9}=\frac{77\left(55 b^{9}+4704 b^{6} \hbar^{2}+110080 b^{3} \hbar^{4}+249856 \hbar^{6}\right)}{125829120} \Omega
\end{aligned}
$$




$$
\begin{aligned}
& b_{10}=\frac{13 b\left(221 b^{9}+24000 b^{6} \hbar^{2}+801024 b^{3} \hbar^{4}+5562368 \hbar^{6}\right)}{1879048192} \Xi \\
& b_{11}=\frac{-11 b^{2}\left(665 b^{9}+89320 b^{6} \hbar^{2}+4051712 b^{3} \hbar^{4}+51793920 \hbar^{6}\right)}{1342177280} \Omega \\
& b_{12}=\frac{-221\left(4641 b^{12}+755040 b^{9} \hbar^{2}+44746240 b^{6} \hbar^{4}+833896448 b^{3} \hbar^{6}+1908572160 \hbar^{8}\right)}{3968549781504} \Xi \\
& b_{13}=\frac{209 b\left(15295 b^{12}+2962960 b^{9} \hbar^{2}+222383616 b^{6} \hbar^{4}+86229749760 b^{3} \hbar^{6}+36359864320 \hbar^{8}\right)}{3350074490880} \Omega \\
& b_{14}=\frac{221 b^{2}\left(1275 b^{12}+289952 b^{9} \hbar^{2}+26885760 b^{6} \hbar^{4}+1040883712 b^{3} \hbar^{6}+10666016768 \hbar^{8}\right)}{6047313952768} \Xi
\end{aligned}
$$

Fourteen terms of the expansion are given above. However, when $n$ in equation (18) greater than 10 and, $b$ is small (e.g. $b=0.1$ ) accuracy up to nine significant figures can be achieved just with the first four terms of the expansion (see Table1). In the literature the symmetric version of the 4th degree potentials are written in the form

$V(x)=x^{2}+\lambda x^{4}$

where $\lambda$ is considered to be small (weak-coupling) and then eigen energies are obtained by perturbative methods. By rescaling the energy $E$ and the coordinate $x$, the potential in (20) can be transformed to the form we have discussed in this section;

$V(x)=x^{4}+b x^{2}$ where $b \sim \frac{1}{\lambda}$.

AEE is very accurate when $|b| \leq 1$, or $|\lambda| \geq 1$ which is corresponding to strong coupling case. On the other hand standard Rayleigh-Schrödinger perturbation expansion is a power series in $\frac{1}{\lambda}$ and it is accurate when $|\lambda| \leq 1$. However, for strongcoupling cases $(|\lambda| \geq 1)$, methôds such as Riccati-Pade method ${ }^{4}$ can also be used. When $x^{3}$ and $x$ terms are non zero in the potential, higher order terms become relatively lengthier. These cases are considered below.

\section{Case II $\quad V(x)=x^{4}+a x^{3}+b x^{2} \quad(a \neq 0, b \neq 0)$}

The eigen energy expansion for this potential is given by

$$
J(E)=n \hbar \approx-\frac{1}{2} \hbar+\sum_{k=0}^{8} b_{k} E^{\left(\frac{3}{4}-\frac{k}{2}\right)}
$$


with

$$
\begin{aligned}
b_{0}= & \mu_{01} \Xi, b_{1}=\left(\mu_{11} a^{2}+\mu_{12} b\right) \Omega, b_{2}=\left(\mu_{21} a^{4}+\mu_{22} a^{2} b+\mu_{23} b^{2}\right) \Xi \\
b_{3}= & \left(\mu_{31} a^{6}+\mu_{32} a^{4} b+\mu_{33} a^{2} b^{2}+\mu_{34} b^{3}+\mu_{35} \hbar^{2}\right) \Omega \\
b_{4}= & -\left(\mu_{41} a^{8}+\mu_{42} a^{6} b+\mu_{43} a^{4} b^{2}+\mu_{44} a^{2} b^{3}+\mu_{45} b^{4}+\mu_{46} a^{2} \hbar^{2}+\mu_{47} b \hbar^{2}\right) \Xi \\
b_{5}= & -\left(\mu_{51} a^{10}+\mu_{52} a^{8} b+\mu_{53} a^{6} b^{2}+\mu_{54} a^{4} b^{3}+\mu_{55} a^{2} b^{4}+\mu_{56} b^{5}+\mu_{57} a^{4} \hbar^{2}+\right. \\
& \left.\mu_{58} a^{2} b h^{2}+\mu_{59} b^{2} \hbar^{2}\right) \Omega \\
b_{6}= & -\left(\mu_{61} a^{12}+\mu_{62} a^{10} b+\mu_{63} a^{8} b^{2}+\mu_{64} a^{6} b^{3}+\mu_{65} a^{4} b^{4}+\mu_{66} a^{2} b^{5}+\mu_{67} b^{6}+\right. \\
& \left.\mu_{68} a^{6} \hbar^{2}+\mu_{69} a^{4} b h^{2}+\mu_{610} a^{2} b^{2} \hbar^{2}+\mu_{611} b^{3} \hbar^{2}+\mu_{612} \hbar^{4}\right) \Xi \\
b_{7}= & \left(\mu_{71} a^{14}+\mu_{72} a^{12} b+\mu_{73} a^{10} b^{2}+\mu_{74} a^{8} b^{3}+\mu_{75} a^{6} b^{4}+\mu_{76} a^{4} b^{5}+\mu_{77} a^{2} b^{6}+\right. \\
& \mu_{78} b^{7}+\mu_{79} a^{8} \hbar^{2}+\mu_{710} a^{6} b h^{2}+\mu_{711} a^{4} b^{2} \hbar^{2}+\mu_{712} a^{2} b^{3} \hbar^{2}+\mu_{713} b^{4} \hbar^{2}+ \\
& \left.\mu_{714} a^{2} \hbar^{4}+\mu_{15} b \hbar^{4}\right) \Omega \\
b_{8}= & \left(\mu_{81} a^{16}+\mu_{82} a^{14} b+\mu_{83} a^{12} b^{2}+\mu_{84} a^{10} b^{3}+\mu_{85} a^{8} b^{4}+\mu_{86} a^{6} b^{5}+\mu_{87} a^{4} b^{6}+\right. \\
& \mu_{88} a^{2} b^{7}+\mu_{89} b^{8}+\mu_{810} a^{10} \hbar^{2}+\mu_{811} a^{8} b h^{2}+\mu_{812} a^{6} b^{2} \hbar^{2}+\mu_{813} a^{4} b^{3} \hbar^{2}+\mu_{814} a^{2} b^{4} \hbar^{2}+ \\
& \left.\mu_{815} b^{5} \hbar^{2}+\mu_{816} a^{4} \hbar^{4}+\mu_{817} a^{2} b \hbar^{4}+\mu_{818} b^{2} \hbar^{4}\right) \Xi
\end{aligned}
$$

and $\mu$ 's are given in the appendix II as rational numbers.

Case III $\quad V(x)=x^{4}+a x^{3}+b x^{2}+c x(a \neq 0, b \neq 0, \& c \neq 0)$

For this potential eigen energy expansion is

$$
J(E)=n \hbar \approx-\frac{1}{2} \hbar+\sum_{k=0}^{5} b_{k} E^{\left(\frac{3}{4}-\frac{k}{2}\right)}
$$

where

$$
\begin{aligned}
b_{0}= & \beta_{01} \Xi, \quad b_{1}=\left(\beta_{11} a^{2}+\beta_{12} b\right) \Omega, \quad b_{2}=\left(\beta_{21} a^{4}+\beta_{22} a^{2} b+\beta_{23} b^{2}+\beta_{24} a c\right) \Xi \\
b_{3}= & \left(\beta_{31} a^{6}+\beta_{32} a^{4} b+\beta_{33} a^{2} b^{2}+\beta_{34} b^{3}+\beta_{35} \hbar^{2}+\beta_{36} a^{3} c+\beta_{37} a b c+\beta_{38} c^{2}\right) \Omega \\
b_{4}= & -\left(\beta_{41} a^{8}+\beta_{42} a^{6} b+\beta_{43} a^{4} b^{2}+\beta_{44} a^{2} b^{3}+\beta_{45} b^{4}+\beta_{46} a^{2} \hbar^{2}+\beta_{47} a^{5} c+\beta_{48} a^{3} b c+\beta_{49} a b^{2} c+\right. \\
& \left.\beta_{410} a^{2} c^{2}+\beta_{411} b c^{2}+\beta_{412} b \hbar^{2}\right) \Xi
\end{aligned}
$$




$$
\begin{aligned}
b_{5}=- & \left(\beta_{51} a^{10}+\beta_{52} a^{8} b+\beta_{53} a^{6} b^{2}+\beta_{54} a^{4} b^{3}+\beta_{55} a^{2} b^{4}+\beta_{56} b^{5}+\beta_{515} a^{4} \hbar^{2}+\right. \\
& \beta_{516} a^{2} b h^{2}+\beta_{517} b^{2} \hbar^{2}+\beta_{57} a^{7} c+\beta_{81} a^{5} b c+\beta_{59} a^{3} b^{2} c+\beta_{514} a b^{3} c+ \\
& \left.\beta_{511} a^{4} c^{2}+\beta_{512} a^{2} b c^{2}+\beta_{513} b^{2} c^{2}+\beta_{514} a c^{3}+\beta_{518} a c \hbar^{2}\right) \Omega
\end{aligned}
$$

and $\beta$ 's are given in the appendix II as rational numbers.

As seen above, when $a \neq 0, b \neq 0 \& c \neq 0$, the coefficients of the higher order terms of AEE become large. However, when $|a| \leq 1, b \leq 1 \& c \leq 1$, only a few terms of the expansion (lower order terms) are needed to get a good approximation for the eigen energies .

\section{HIGHER DEGREE POLYNOMIAL POTENTIALS}

Higher degree polynomial potentials (degree $>4$ ) can be treated the same way as the 4th degree case. However, AEE formulae become large if all the terms of the general polynomial potentials are included. (As an example, for the sixth order, general polynomial potential contains powers $x^{5}, x^{4}, x^{3}, x^{2}$ and $x$ in addition to the $x^{6}$ term) Therefore, only the harmonic term and the leading term of the polynomial are included for the present study. Nevertheless, there is no restriction on treating the general polynomial potential of any degree with all the terms included.

Potential 1. $\left(V(x)=x^{6}+b x^{2}\right.$

As in the case of $4^{\text {th }}$ degree polynomial potential, $6^{\text {th }}$ degree polynomial potentials, which have been treated in the literature, are usually of the form $V(x)=\frac{1}{2} x^{2}+\lambda x^{6}$ where $|\lambda|$ is small.

Here we present AEE for the potential $V(x)=x^{6}+b x^{2} . \quad \lambda$ and $b$ are related as before; $b \sim \frac{1}{\lambda}$. After using recurrence relations, AEE is obtained

$$
\begin{gathered}
J(E)=n \hbar \approx-\frac{1}{2} \hbar+b_{0} E^{\frac{4}{6}}+b_{1}+b_{2} E^{-\frac{4}{6}}+b_{3} E^{-\frac{8}{6}}+b_{4} E^{-\frac{16}{6}}+b_{5} E^{-\frac{20}{6}}+b_{6} E^{-\frac{28}{6}}+ \\
b_{7} E^{-\frac{32}{6}}+b_{8} E^{-\frac{40}{6}}
\end{gathered}
$$


where

$b_{0}=\frac{\Gamma\left[\frac{1}{6}\right]}{4 \sqrt{\pi} \Gamma\left[\frac{2}{3}\right]}, b_{1}=-\frac{b}{6}, b_{2}=\frac{\left(b^{2}-5 \hbar^{2}\right)}{12} \frac{\Gamma\left[\frac{5}{6}\right]}{\sqrt{\pi} \Gamma\left[\frac{1}{3}\right]}$,

$b_{3}=\frac{b(b-3 \hbar)(b+3 \hbar)}{648} \frac{\Gamma\left[\frac{1}{6}\right]}{\sqrt{\pi} \Gamma\left[\frac{2}{3}\right]}$

$b_{4}=-\frac{5 b(b-3 \hbar)(b+3 \hbar)\left(b^{2}-29 \hbar^{2}\right)}{3888} \frac{\Gamma\left[\frac{5}{6}\right]}{\sqrt{\pi} \Gamma\left[\frac{1}{3}\right]}$

$b_{5}=-\frac{\left.49 b^{6}-2835 b^{4} \hbar^{2}+44163 b^{2} \hbar^{4}-77025 \hbar^{6}\right)}{1049760} \frac{\Gamma\left[\frac{1}{6}\right]}{\sqrt{\pi} \Gamma\left[\frac{2}{3}\right]}$

$b_{6}=\frac{11\left(55 b^{8}-6020 b^{6} \hbar^{2}+237762 b^{4} \hbar^{4}-3133140 b^{2} \hbar^{6}+5678175 \hbar^{8}\right)}{8817984} \frac{\Gamma\left[\frac{5}{6}\right]}{\sqrt{\pi} \Gamma\left[\frac{1}{3}\right]}$

$b_{7}=\frac{13 b(b-3 \hbar)(b+3 \hbar)\left(91 b^{6}-12033 b^{4} \hbar^{2}+608769 b^{2} \hbar^{4}-10608795 \hbar^{6}\right)}{408146688} \frac{\Gamma\left[\frac{1}{6}\right]}{\sqrt{\pi} \Gamma\left[\frac{2}{3}\right]}$

$b_{8}=-\frac{187 b(b-3 \hbar)(b+3 \hbar)\left(17 b^{8}-3532 b^{6} \hbar^{2}+324990 b^{4} \hbar^{4}-14499036 b^{2} \hbar^{6}+238937625 \hbar^{8}\right)}{612220032} \times$ $\frac{\Gamma\left[\frac{5}{6}\right]}{\sqrt{\pi} \Gamma\left[\frac{1}{3}\right]}$

(26) 
The comparison of the exact eigen energies with the AEE eigen values are shown in Table 2. Only seven terms of AEE are used. $b$ is taken as 0.1. However, for $b>1$ (i.e. $\lambda<1$ ) the AEE expression still produces reasonably good results. (see Table 3.).

Next we present AEE expansions for the potentials $V(x)=x^{8}+b x^{2}$ and $V(x)=x^{10}+b x^{2}$. The number of terms given below are quite adequate for obtaining accurate eigen values from the AEE when $|b| \leq 1$.

Potential 2. $\left(V(x)=x^{8}+b x^{2}\right)$

The AEE expansion for this potential is

$$
\begin{gathered}
J(E)=n \hbar \approx-\frac{1}{2} \hbar+b_{0} E^{\frac{5}{8}}+b_{1} E^{-\frac{1}{8}}+b_{2} E^{-\frac{5}{8}}+b_{3} E^{-\frac{7}{8}}+b_{4} E^{-\frac{11}{8}}+b_{5} E^{-\frac{13}{8}}+b_{6} E^{-\frac{15}{8}}+b_{7} E^{-\frac{17}{8}} \\
+b_{8} E^{-\frac{19}{8}}+b_{9} E^{-\frac{21}{8}}+b_{10} E^{-\frac{23}{8}}+b_{11} E^{-\frac{25}{8}}
\end{gathered}
$$

where

$$
\begin{aligned}
& b_{0}=\frac{\Gamma\left[\frac{1}{8}\right]}{5 \sqrt{\pi} \Gamma\left[\frac{5}{8}\right]}, b_{1}=-\frac{b \Gamma\left[\frac{3}{8}\right]}{8 \sqrt{\pi} \Gamma\left[\frac{7}{8}\right]}, \quad b_{2}=-\frac{7 \hbar^{2} \Gamma\left[\frac{7}{8}\right]}{12 \sqrt{\pi} \Gamma\left[\frac{3}{8}\right]}, b_{3}=\frac{b^{2} \Gamma\left[\frac{5}{8}\right]}{16 \sqrt{\pi} \Gamma\left[\frac{1}{8}\right]}, \\
& b_{4}=-\frac{5 b \hbar^{2}}{256} \frac{\Gamma\left[\frac{1}{8}\right]}{\sqrt{\pi} \Gamma\left[\frac{5}{8}\right]}, \quad b_{5}=\frac{5 b^{3}}{384} \frac{\Gamma\left[\frac{7}{8}\right]}{\sqrt{\pi} \Gamma\left[\frac{3}{8}\right]}, b_{6}=-\frac{931 h^{4}}{2304} \frac{\Gamma\left[\frac{5}{8}\right]}{\sqrt{\pi} \Gamma\left[\frac{1}{8}\right]}, \\
& b_{7}=-\frac{51 b^{2} \hbar^{2}}{4096} \frac{\Gamma\left[\frac{3}{8}\right]}{\sqrt{\pi} \Gamma\left[\frac{7}{8}\right]} \\
& b_{8}=-\frac{11 b^{4}}{32768} \frac{\Gamma\left[\frac{1}{8}\right]}{\sqrt{\pi} \Gamma\left[\frac{5}{8}\right]}, b_{9}=-\frac{5005 b h^{4}}{6144} \frac{\Gamma\left[\frac{7}{8}\right]}{\sqrt{\pi} \Gamma\left[\frac{3}{8}\right]}, b_{10}=\frac{1015 b^{3} h^{2}}{12288} \frac{\Gamma\left[\frac{5}{8}\right]}{\sqrt{\pi} \Gamma\left[\frac{1}{8}\right]}
\end{aligned}
$$




$$
b_{11}=\frac{17\left(9 b^{5}+16828 \hbar^{6}\right)}{1310720} \frac{\Gamma\left[\frac{3}{8}\right]}{\sqrt{\pi} \Gamma\left[\frac{7}{8}\right]}
$$

Potential 3. $\left(V(x)=x^{10}+b x^{2}\right)$

For the 10th degree polynomial potential, the first ten terms of AEE are given as

$$
\begin{gathered}
J(E)=n \hbar \approx-\frac{1}{2} \hbar+b_{0} E^{\frac{3}{5}}+b_{1} E^{-\frac{1}{5}}+b_{2} E^{-\frac{3}{5}}+b_{3} E^{-\frac{7}{5}}+b_{4} E^{-\frac{9}{5}}+b_{5} E^{-\frac{11}{5}}+b_{6} E^{-\frac{13}{5}}+b_{7} E^{-\frac{17}{5}} \\
+b_{8} E^{-\frac{19}{5}}+b_{9} E^{-\frac{21}{5}}
\end{gathered}
$$

where

$b_{0}=\frac{\Gamma\left[\frac{1}{10}\right]}{6 \sqrt{\pi} \Gamma\left[\frac{3}{5}\right]}, \quad b_{1}=-\frac{b \Gamma\left[\frac{3}{10}\right]}{10 \sqrt{\pi} \Gamma\left[\frac{4}{5}\right]}, \quad b_{2}=-\frac{3 \hbar^{2} \Gamma\left[\frac{9}{10}\right]}{4 \sqrt{\pi} \Gamma\left[\frac{2}{5}\right]}$

$b_{3}=-\frac{7 b \hbar^{2} \Gamma\left[\frac{1}{10}\right]}{300 \sqrt{\pi} \Gamma\left[\frac{3}{5}\right]}, \quad b_{4}=\frac{\left(8 b^{3}-483 \hbar^{4}\right) \Gamma\left[\frac{7}{10}\right]}{600 \sqrt{\pi} \Gamma\left[\frac{1}{5}\right]}$

$b_{5}=-\frac{23 b^{2} h^{2} \Gamma\left[\frac{3}{10}\right]}{1000 \sqrt{\pi} \Gamma\left[\frac{4}{5}\right]}, b_{6}=\frac{b\left(2 b^{3}-777 \hbar^{4}\right) \Gamma\left[\frac{9}{10}\right]}{500 \sqrt{\pi} \Gamma\left[\frac{2}{5}\right]}, b_{7}=\frac{7 b^{2}\left(12 b^{3}-16345 \hbar^{4}\right) \Gamma\left[\frac{1}{10}\right]}{750000 \sqrt{\pi} \Gamma\left[\frac{3}{5}\right]}$

$b_{8}=\frac{7 b h^{2}\left(110 b^{3}-34599 \hbar^{4}\right) \Gamma\left[\frac{7}{10}\right]}{10000 \sqrt{\pi} \Gamma\left[\frac{1}{5}\right]}$ 


$$
b_{9}=\frac{11\left(672 b^{6}-2324560 b^{3} h^{4}+175050105 \hbar^{8}\right) \Gamma\left[\frac{3}{10}\right]}{105000000 \sqrt{\pi} \Gamma\left[\frac{4}{5}\right]}
$$

As described earlier, AEE formulae can be derived for polynomial of any degree using the recurrence relations. Using computer algebra packages such as MATHEMATICA, the integrals can be evaluated and $\Gamma$ functions are simplified in the same routine. One example is given in Appendix I. for the potential $V(x)=x^{6}+b x^{2}$. We have given a short MATHEMATICA code which will not only derive coefficients of the AEE using recurrence relations, but also evaluate the integrals and simplifies the $\Gamma$ functions in one go.

\section{DISCUSSION}

In this paper we presented AEEs for polynomial potentials, which give accurate eigen energies specially for higher excited states. By inverting AEEs, one could obtain eigen energies. The recurrence relations derived in section 2. can be used with any even degree polynomial potential and all the integrals in AEE can be evaluated in terms of $\Gamma$ functions analytically. All the non zero integrals have the general form

$$
\eta_{N}(n, m)=\int_{\gamma} \frac{y^{n}}{\left(1-y^{2 N}\right)^{m+\frac{1}{2}}} d y=\frac{\Gamma\left(\frac{1}{2}-m\right) \Gamma\left(1+\frac{1}{2 N}+\frac{n}{2 N}\right)}{\pi(1+n) \Gamma\left(\frac{1}{2}+\frac{1}{2 N}-m+\frac{n}{2 N}\right)}
$$

except for one, which has the form.

$$
\int_{\gamma} \frac{y^{2 N-1}}{\left(1-y^{2 N}\right)} d y
$$

where $2 N$ is the degree of the polynomial potential.

There are some resemblance between AEE terms derived for polynomial potentials here and the WKB terms derived for $x^{2 N}$ by Bender et $\mathrm{al}^{1}$. However, for polynomial potentials, in addition to the terms due to $x^{2 N}$ part of the potential (WKB type), there are terms which contain coefficients of the polynomial potential as well.

In this paper we considered only the polynomial potentials of even degree. Polynomial potentials of odd degree can be handled the same way as in the even degree case (e.g. PT invariant potential $\left.v(x)=i x^{3}+b x^{2}\right)$. However, for odd degree potentials, the turning points which should be included in the contour integration are not as obvious as in the case of even degree. Also note that AEE is meaningful only 
when the potential possesses bound states. On the other hand, even degree polynomial potentials always possess bound states.

AEE produces very accurate bound state eigen values when the coefficients of the potentials are less than unity. However, all the polynomial potentials we studied so far produced quite accurate energy eigen values even for the coefficients greater than unity. 
Table 1. Comparison between calculated energy eigen values by AEE with four terms and $E_{\text {Exact }}$ which is obtained by matrix diagonalization method with harmonic oscillator basis functions for the potential $v(x)=x^{4}+b x^{2} \quad(\mathrm{~b}=0.1, \mathrm{~h}=1.0)$

\begin{tabular}{|c|c|c|}
\hline$n$ & $E_{A E E}$ & $E_{\text {Exact }}$ \\
\hline 0 & 1.021970834631 & 1.096224366160 \\
\hline 1 & 3.898059013343 & 3.889264230279 \\
\hline 2 & 7.579582959880 & 7.57970432951 \\
\hline 3 & 11.800122705856 & 11.8000665467 \\
\hline 4 & 16.445500588092 & 16.4455187665 \\
\hline 5 & 21.448373304913 & 21.44840638544 \\
\hline 6 & 26.763253767437 & 26.76329054978 \\
\hline 7 & 32.356924280183 & 32.35696056521 \\
\hline 8 & 38.203847374396 & 38.20388172575 \\
\hline 9 & 44.283657210779 & 44.28368921279 \\
\hline 10 & 50.579658893960 & 50.57968853111 \\
\hline 20 & 123.110105565756 & 123.1101204989 \\
\hline 30 & 208.891239872221 & 208.8912491422 \\
\hline 40 & 304.708185378200 & 304.7081918800 \\
\hline 50 & 408.796731799018 & 408.7967367059 \\
\hline 100 & 1022.449599822648 & 1022.449601827 \\
\hline
\end{tabular}


Table 2. Comparison between calculated energy eigen values by AEE with four terms and $E_{\text {Exact }}$ which is obtained by matrix diagonalization method with harmonic oscillator basis functions for the potential $v(x)=x^{6}+b x^{2} \quad(\mathrm{~b}=0.1, \hbar=1.0)$

\begin{tabular}{|c|c|c|}
\hline$n$ & $E_{A E E}$ & $E_{\text {Exact }}$ \\
\hline 0 & 1.1754106584 & 1.1499943198 \\
\hline 1 & 4.4101778724 & 4.3764304659 \\
\hline 2 & 9.1634248118 & 9.1653332985 \\
\hline 3 & 15.0413687683 & 15.0415571713 \\
\hline 4 & 21.8344446110 & 21.8344312332 \\
\hline 5 & 29.4325487695 & 29.4325477012 \\
\hline 6 & 37.7575253302 & 37.7575254279 \\
\hline 7 & 46.7503372639 & 46.7503373421 \\
\hline 8 & 56.3644275631 & 56.3644274972 \\
\hline 9 & 66.5618394905 & 66.5618395285 \\
\hline 10 & 77.3108479462 & 77.3108480191 \\
\hline 20 & 210.5398511446 & 210.5398510955 \\
\hline 30 & 381.8836788818 & 381.8836788443 \\
\hline 40 & 584.1961076325 & 584.1961077105 \\
\hline 50 & 813.3023130233 & 813.3023130293 \\
\hline 100 & 2282.6858971094 & 2282.6858971096 \\
\hline
\end{tabular}


Table 3. Comparison between calculated energy eigen values by AEE with four terms and $E_{\text {Exact }}$ which is obtained by matrix diagonalization method with harmonic oscillator basis functions for the potential $v(x)=x^{6}+b x^{2} \quad(\mathrm{~b}=5, \hbar=1.0)$

\begin{tabular}{|c|l|l|}
\hline$n$ & \multicolumn{1}{|c|}{$E_{A E E}$} & \multicolumn{1}{|c|}{$E_{\text {Exact }}$} \\
\hline 0 & 2.2608592319 & 2.35860354969736 \\
\hline 1 & 7.4378040804 & 7.42797837403537 \\
\hline 2 & 13.2929197836 & 13.2929645882579 \\
\hline 3 & 20.0161803598 & 20.0162298834247 \\
\hline 4 & 27.5313785523 & 27.5313788612371 \\
\hline 5 & 35.7698383151 & 35.7698374668921 \\
\hline 6 & 44.6760278389 & 44.6760271790176 \\
\hline 7 & 54.2047974187 & 54.2047969685539 \\
\hline 8 & 64.3187642186 & 64.318764144693 \\
\hline 9 & 74.9864095596 & 74.9864090282413 \\
\hline 10 & 86.1807319656 & 86.1807317393121 \\
\hline 20 & 223.0169538574 & 223.016953932628 \\
\hline 30 & 397.1373374200 & 397.137336922934 \\
\hline 40 & 601.7934935755 & 601.793493563877 \\
\hline 50 & 832.9660098585 & 832.966010852819 \\
\hline 100 & 2310.4639746067 & 2310.46397455999 \\
\hline & & \\
\hline
\end{tabular}




\section{Appendix I}

Here we present a short MATHEMATICA code which derives coefficients of the AEE using recurrence relations, Also it evaluates the integrals and simplifies the $\Gamma$ functions to give simplified individual terms of AEE. Please disregard terms in bold. $a_{m}(y, \varepsilon)$ in equation (13) is denoted here as $\mathrm{y}\left[\mathrm{m}, \mathrm{y}, \mathrm{h} \_\right]$.

(* POTENTIAL $V(x)=x^{6}+b x^{2} *$ )

(*Using recurrence relations in equation (10) $a_{m}(y, \varepsilon)$ are derived*)

In[1]:=

$\mathrm{V} 0\left[\mathrm{x}_{-}\right]:=\mathrm{x}^{\wedge} 6$;

$\mathrm{y}\left[0, \mathrm{x}_{\text {_h_ }}\right]:=(1-\mathrm{V} 0[\mathrm{x}])^{\wedge}(1 / 2)$;

$\mathrm{y}\left[1, \mathrm{x}_{-}, \mathrm{h} \_\right]:=0$;

$\mathrm{y}\left[2, \mathrm{x}_{-}, \mathrm{h} \_\right]:=0$;

$\mathrm{y}\left[3, \mathrm{x}_{\text {, }} \mathrm{h}\right] \mathrm{]}=0$;

$\mathrm{y}\left[4, \mathrm{x} \_\mathrm{h} \_\right]:=-\left(1 /(2 * y[0, \mathrm{x}, \mathrm{h}]) *\left(\mathrm{~b}^{*} \mathrm{x}^{\wedge} 2+\mathrm{h} / \mathrm{I} * \mathrm{D}[\mathrm{y}[0, \mathrm{x}, \mathrm{h}], \mathrm{x}]\right)\right)$;

$y\left[n \_, x_{-}, h \_\right]:=-(1 /(2 * y[0, x, h]) *(\operatorname{Sum}[y[i, x, h] * y[n-i, x, h],\{i, 1, n-1\}]+h / I * D[y[$

$\mathrm{n}-4, \mathrm{x}, \mathrm{h}], \mathrm{x}]))$;

(*In order to simplify Gamma functions automatically, here we apply recurrence relations such as $\Gamma[n+1]=n \Gamma[n] *)$

$\operatorname{In}[8]:=$

ga[n_]:=(

If[n>1, $=(n-1) * g a[n-1]$,

If $[\mathrm{n}<0, \mathrm{q}=\mathrm{ga}[\mathrm{n}+1] / \mathrm{n}, \mathrm{q}=\mathrm{Gamma}[\mathrm{n}]]]$;

q);

\section{In[81]:= (*Here we obtain $\left.b_{8} *\right)$}

$\mathrm{nn}=8$;

(*Keep $y[8, x, h]$ and $y[8, x,-h]$ in the memory for the next calculation (i.e. when $n n=9)$.

This will reduce calculation time drastically *)

$\mathrm{y}[\mathrm{nn}, \mathrm{x}, \mathrm{h}]=$ Expand[y[nn,x,h]];

$\mathrm{y}[\mathrm{nn}, \mathrm{x},-\mathrm{h}]=$ Expand[y[nn,x,-h]];

(*To eliminate terms with zero contributions and some algebraic manipulations to make sure that integrals will not blow up *)

$\mathrm{y} 1[\mathrm{nn}, \mathrm{y}]=\operatorname{Expand}\left[((\mathrm{y}[\mathrm{nn}, \mathrm{x}, \mathrm{h}]+\mathrm{y}[\mathrm{nn}, \mathrm{x},-\mathrm{h}]) / 2) /\left(1-\mathrm{x}^{\wedge} 6\right)^{\wedge} \mathrm{t}\right] / .\{\mathrm{x}->\mathrm{y}\}$; 
b1[nn,y_]=Integrate[y1[nn,y],y];

b2[nn]=Simplify[(b1[nn,1]-b1[nn,-1])/Pi];

Factor[Expand[b2[nn]]/.\{t->0,Gamma->ga $\}]$

(* ----------------------Results

\section{Out[88]=}

$\left(* b_{8}\right.$ is $\left.*\right)$

22

5

(b - 5 h ) Gamma[-]

6

1

12 Sqrt[Pi] Gamma[-]

3

\section{Appendix II}

$$
V(x)=x^{4}+a x^{3}+b x^{2}
$$

The coefficients of the AEE expansion in equation (22) are given below.

$$
\begin{aligned}
& \mu_{01}=\frac{1}{3} \\
& \mu_{11}=\frac{3}{8} \quad \mu_{12}=-1 \\
& \mu_{22}=\frac{15}{2048} \quad \mu_{22}=-\frac{5}{128} \quad \mu_{22}=\frac{1}{32} \\
& \mu_{31}=\frac{77}{16384} \quad \mu_{32}=-\frac{77}{2048} \quad \mu_{33}=\frac{21}{256} \quad \mu_{34}=-\frac{1}{32} \quad \mu_{35}=-\frac{1}{4} \\
& \mu_{41}=\frac{663}{8388608} \quad \mu_{42}=-\frac{221}{262144} \quad \mu_{43}=\frac{195}{65536} \mu_{44}=-\frac{15}{4096} \\
& \mu_{46}=-\frac{1}{512} \quad \mu_{47}=\frac{1}{192}
\end{aligned}
$$




$$
\begin{gathered}
\mu_{51}=\frac{43263}{335544320} \quad \mu_{52}=-\frac{14421}{8388608} \quad \mu_{53}=\frac{4389}{524288} \quad \mu_{54}=-\frac{1155}{65536 a} \\
\mu_{55}=\frac{231}{16384} \quad \mu_{56}=-\frac{21}{10240} \quad \mu_{57}=-\frac{63}{8192} \quad \mu_{58}=\frac{21}{512} \\
\mu_{59}=-\frac{5}{128} \quad \mu_{62}=\frac{160225}{3221225472} \quad \mu_{63}=-\frac{82875}{268435456} \\
\mu_{61}=-\frac{160225}{51539607552} \quad \mu_{65}=-\frac{5525}{4194304} \quad \mu_{66}=\frac{195}{262144} \\
\mu_{64}=\frac{7735}{8388608} \quad \mu_{68}=\frac{325}{1048576} \quad \mu_{69}=-\frac{325}{131072} \\
\mu_{67}=-\frac{5}{65536} \quad \mu_{611}=-\frac{5}{2048} \quad \mu_{612}=-\frac{11}{1536} \\
\mu_{610}=\frac{275}{49152} \quad \mu^{264}
\end{gathered}
$$

$$
\begin{array}{cc}
\mu_{71}=\frac{853461}{137438953472} & \mu_{72}=-\frac{1991409}{17179869184} \quad \mu_{73}=\frac{9388}{1073741} \quad \mu_{76}=-\frac{30723}{4194304} \\
\mu_{74}=-\frac{908523}{268435456} & \mu_{75}=\frac{235543}{33554432} \quad \mu_{78}=-\frac{77}{327680} \quad \mu_{79}=-\frac{30723}{33554432} \\
\mu_{77}=\frac{1617}{524288} & \mu_{711}=-\frac{9163}{262144} \quad \mu_{712}=\frac{735}{16384} \\
\mu_{710}=\frac{10241}{1048576} & \mu_{714}=\frac{105}{4096} \mu_{715}=-\frac{35}{512}
\end{array}
$$

$$
\begin{gathered}
\mu_{81}=-\frac{168273225}{985162418487296} \quad \mu_{82}=\frac{56091075}{15393162788864} \quad \mu_{83}=-\frac{17784975}{549755813888} \\
\mu_{84}=\frac{5287425}{34359738368} \quad \mu_{85}=-\frac{7210125}{17179869184} \quad \mu_{86}=\frac{348075}{536870912} \\
\mu_{87}=-\frac{69615}{134217728} \quad \mu_{88}=\frac{9945}{58720256} \quad \mu_{89}=-\frac{585}{58720256}
\end{gathered}
$$




$$
\begin{gathered}
\mu_{810}=\frac{149175}{4294967296} \quad \mu_{811}=-\frac{248625}{536870912} \quad \mu_{812}=\frac{76245}{33554432} \\
\mu_{813}=-\frac{20475}{4194304} \quad \mu_{814}=\frac{4275}{1048576} \quad \mu_{815}=-\frac{85}{131072} \\
\mu_{816}=-\frac{1755}{1048576} \quad \mu_{817}=\frac{585}{65536} \quad \mu_{818}=-\frac{173}{16384} \\
\mathbf{V ( x )}=\mathbf{x}^{4}+\mathbf{a x}^{3}+\mathbf{b x}^{2}+\mathbf{c x}
\end{gathered}
$$

The coefficients of the AEE expansion in equation (24) are given below.

$$
\begin{aligned}
& \beta_{01}=\frac{1}{3} \\
& \beta_{11}=\frac{3}{8} \quad \beta_{12}=-1 \\
& \beta_{21}=\frac{15}{2048} \quad \beta_{22}=-\frac{5}{128} \quad \beta_{23}=\frac{1}{32} \quad \beta_{24}=\frac{1}{16} \\
& \beta_{31}=\frac{77}{16384} \beta_{32}=-\frac{77}{2048} \beta_{33}=\frac{21}{256} \quad \beta_{34}=\frac{1}{32} \quad \beta_{35}=-\frac{1}{4} \quad \beta_{36}=\frac{7}{128} \\
& \beta_{41}=\frac{638}{8388608}=-\frac{3}{16} \quad \beta_{38}=\frac{1}{8} \quad \beta_{42}=-\frac{221}{262144} \quad \beta_{43}=\frac{195}{65536} \beta_{44}=-\frac{15}{4096} \\
& \beta_{45}=\frac{5}{6144} \quad \beta_{46}=-\frac{1}{512} \quad \beta_{47}=\frac{39}{32768} \\
& \beta_{410}=\frac{5}{1024}
\end{aligned}
$$




$$
\begin{aligned}
& \beta_{513}=-\frac{21}{512} \beta_{514}=\frac{231}{16384} \quad \beta_{515}=-\frac{63}{1024} \quad \beta_{516}=\frac{9}{256} \\
& \beta_{517}=\frac{3}{128} \quad \beta_{518}=-\frac{3}{64}
\end{aligned}
$$

\section{REFERENCES}

[1] C. M. Bender, K. Olaussen, and P. S. Wang, Phys. Rev. D 16, 1740. (1977)

[2] M. Robnik and L. Salasnich, J. Phys. A:Math. Gen. 30, 1719 (1997)

[3] Andre Voros, J. Phys A: Math. Gen. 32, 5993 (1999)

[4] F M Fernandez and R Guardiola 1993 J. Phys. A: Math. Gen. 26, 7169

[5] B Bacus, Y Meurice, and A Soemadi J. Phys. A: Math. Gen. 28, (1995) L381

[6] Marko Robnik and Valery G Romanovski J. Phys. A: Math. Gen. 33, (2000) 5093

[7] G A Dobrovolsky and Tutik J. Phys. A: Math. Gen. 33, (2000) 6593

[8] Gabriel Alvarez and Carmen Casares J. Phys. A: Math. Gen. 33, (2000) 2499

[9] Pravat Kumar Patnaik Phy Rev D 35, (1987) 1234

[10] A D Speliotopous J. Phys. A: Math. Gen. 33, (2000) 3809

[11] A V Sergev J. Phys. A: Math. Gen. 28, (1995) 4157

[12] L D Salem and R Montemayor Phy Rev A 43,(1991) 1169

[13] G A Dobrovolsky and Tutik J. Phys. A: Math. Gen. 32, (1999) 563

[14] Andre Voros, J. Phys A: Math. Gen. 27, 4653 (1994)

[15] I A Ivanov J. Phys A: Math. Gen. 31, 6995 (1998)

[16] Asiri Nanayakkara, Phy Lett A. 289, 39 (2001) 